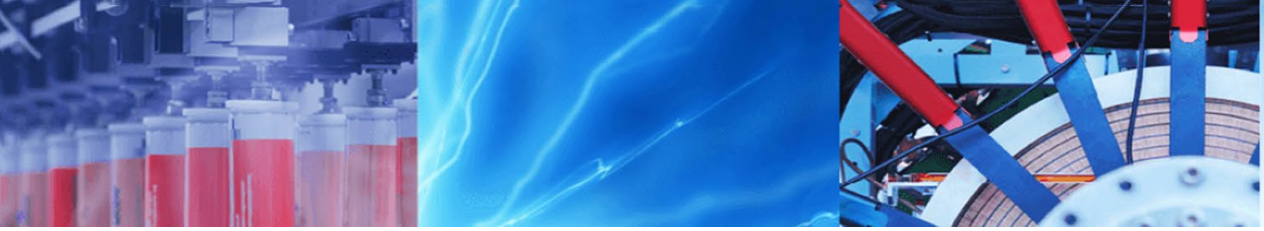

Research Article

\title{
Energy efficient wireless sensor network using optimum hops and virtual MIMO technique
}

\author{
Manish Kumar Singh ${ }^{1,2}$ D Syed Intekhab Amin ${ }^{2}$
}

Received: 1 May 2020 / Accepted: 18 August 2020 / Published online: 28 August 2020

(c) Springer Nature Switzerland AG 2020

\begin{abstract}
The virtual multiple-input multiple-output (V-MIMO) configuration provide the reliable broadband wireless communication that raises the data rate and reduce the energy consumption, for long range communication in the wireless sensor network (WSN). Generally in the WSN, the source node does not have sufficient power and transmission range to transmit data packets directly to the sink node. Therefore, the multihop technique is necessary to transmit data from source node to sink node. In this paper, multihop V-MIMO technique is used for long range communication in the WSN. In this technique, the source-to-sink route divides into many optimum hops, following which the $2 \times 2 \mathrm{~V}-\mathrm{MIMO}$ technique is applied to all the hops. The simulation results show that for long range communication, the multihop MIMO technique provides better energy saving than the MIMO technique or multihop single-input single-output (SISO) technique in a WSN, while satisfying the throughput and delay. The paper also presents the effect of path loss exponent on energy consumption. The simulation results clearly indicate that the energy consumption increases with an increase in the path loss exponent in the SISO, MIMO, single and multihop communication techniques. The result reveals that the network reliability is inversely proportional to the number of hops.
\end{abstract}

Keywords Wireless sensor network · Single input single output (SISO) - Virtual multiple input multiple output $(\mathrm{V}$-MIMO) $\cdot$ Cluster head $(\mathrm{CH}) \cdot$ Sensor nodes

\section{Introduction}

A wireless sensor network (WSN) has various potential applications like in environmental monitoring, medical care, battlefield surveillance, wildlife monitoring, etc. [1]. In some applications, the replacement of batteries is a very difficult task. Therefore, power saving is a very important issue in WSN [2, 3]. Clustering technique is generally used to design energy efficient WSNs. It divides the WSN into various clusters. Each cluster consists of many nodes and one or more cluster head $(\mathrm{CH})$. $\mathrm{CHs}$ collect and aggregate data from nodes and transmit it to their base station (BS). Selection process of cluster head within the system can help to maximize network performance as cluster head is responsible for various energy consuming tasks [4]. Sleep and wake technique with Time division multiple access (TDMA) scheme is used in WSN because of low power consumption and collision free data transmission [5]. In this technique, the radio of every sensor node remains switch off mode and it comes in switch on mode in its assigned broadcast time. The receiver of cluster head should be in switch on mode to collect data from its members [6]. The direct data transfer from the source node to sink node may not be possible due to limited signal amplification of the sensor nodes. Therefore, multihop communication is necessary for long distance communication in WSN. In single

$\triangle$ Manish Kumar Singh, mks.manish1983@gmail.com | 'Department of Electronics and Communication Engineering, KIET Group of Institutions, Ghaziabad, Uttar Pradesh 201206, India. ${ }^{2}$ Department of Electronics and Communication Engineering, Faculty of Engineering and Technology, Jamia Millia Islamia, New Delhi 110025, India. 
hop communication, the source node directly communicates with the sink nodes without any relay nodes, whereas in multihop communication, a relay node is necessary for forwarding the source data to the sink [7]. Therefore, sensor nodes are responsible for configuring the WSNs. Additionally; WSNs have numerous advantages such as low cost, flexibility, rapid deployment, etc. In a WSN, either less large hops or more small hops is required to transmit data from source node to sink. Each procedure long or short hops has its own benefits. Transmitting information over long hops reduce the cost because the least number of sensor nodes are used in data routing. On the other hand, transmitting information over short hops reduce the transmission energy that is proportional to the transmission distance [8]. When we need minimum power consumption between two nodes, the short hop strategy is preferred. But the reception cost cannot be ignored. Hence, there are certain distances between the source and destination within which direct communication is considered a better choice. Thus, using the multihop technique is not the best method in every instance. When we form energy efficient WSN, the number of hops increases with an increase in the communication distance. Various researches on the WSN have generally focused on either optimizing the length of hop or minimizing transmitting power, while keeping the other parameter as constant. Therefore, by choosing the optimal hop length, unnecessary power consumption can be saved due to reducing the intermediate sensor nodes between the source and the sink [9].

The V-MIMO technique provides high data rates without increasing transmission energy [10]. For long distance communication, $\mathrm{V}$-MIMO needs less transmission energy than the SISO technique [2, 3]. In the V-MIMO technique, some nodes are chosen as cooperative nodes, which are used to form a V-MIMO structure. The selection of cooperative nodes depends on channel conditions, residual energy, geographic location and distance between the nodes. Several virtual MIMO topologies can be configured in a WSN, such as $1 \times 2$ single-input multiple-output (SIMO), $2 \times 2 \mathrm{MIMO}$ and $2 \times 1$ multiple-input single-output (MISO), etc. The energy consumption of single hop SISO, MIMO, SIMO and MISO are analyzed $[7,11]$. The source node tries to determine the best energy efficient configuration for data transmission among SISO, V-MISO, V-MIMO, or V-SIMO [12]. Space time block coding (STBC) is used in a V-MIMO technique [7].

Energy consumption is a major problem in nodes as compared to sink because nodes are deployed at remotely location, and the frequent replacement of the battery is not an easy task [13]. The lessening in signal power density between the transmitter and receiver route is known as path loss. The transmitted signal can be reflected, scattered and diffracted due to obstacles. Thus, modeling of the path loss is not an easy task [14]. In [8], the authors studied that data from the source node to the destination node may send in either long or short hops. Thus, optimizing the hop length, can save energy as well as improve the lifetime of the WSN. In [14], the authors found that the supremacy of the multi hop method depends on the reception cost and the distance between the source and the sink.

The remainder of the paper is organized as follows: In Sect. 2, problem description and proposed work is discussed. Network model is described in Sect. 3. Sections 4 and 5 , present the energy consumption and reliability analysis of the proposed technique. Section 6 presents simulation results and discussion. Finally, paper is concluded in Sect. 7.

\section{Problem description and proposed work}

A Wireless sensor network (WSN) generally uses single antenna and relaying technique for long distance data transmission and reception that is called a multihop SISO configuration. It is not energy efficient for long distance communication and also provides low data rate. The V-MIMO technique is used to increase the data rate and reduce energy consumption for long distance communication in WSN [10]. But, WSN covers a large area. Therefore, multihop SISO or V-MIMO is not suitable for very long distance communication in every circumstance. SISO and V-MIMO techniques can determine optimum hops between source and sink that reduce the energy consumption and hop count in the WSN. Energy efficiency depends upon the path loss between transmitter and receiver. Hence, modeling of path loss is important in a single hop and multihop energy efficient wireless sensor network. These are not addressed in the existing research work.

A multihop technique can reduce the energy consumption of the overall route with good network connectivity using the SISO, MISO, SIMO or MIMO configurations. Therefore, multihop V-MIMO technique is used in this paper. We first compare energy consumption per bit in the single hop, multihop, SISO, V-MIMO transmission techniques for some fixed distance in the WSN. This work is different from previous research work because we determine the number of optimum hops between the source and the destination path for various fixed distances in SISO and V-MIMO techniques that consume lowest energy on that specific distance in the WSN. Then, energy consumption of MIMO, SISO and optimum hop MIMO techniques is compared. Number of hops can be properly selected in the WSN on the basis of cost or energy consumption according to the requirement. This paper also discusses the effect of energy consumption per bit for single hop, multihop, SISO and 
MIMO communication techniques on different values of the path loss exponent in short and long distance communication. The effect of hop count on reliability of network is also discussed for better guideline. The MATLAB Lab simulation tool has been used for evaluation of the experimental results.

\section{Network model}

In Fig. 1, the distance between the source to the destination node is $d$. In this figure, the route from the source to the destination is divided into 1 hop, 2 hop and 3 hop respectively. Figure 2 shows the network topology of the V-MIMO, V-SIMO and V-MISO techniques.

Figure 3 shows the two hop homogeneous network using the V-MIMO scheme, where the first hop is from the source to the intermediate node while the second hop is from the intermediate node to the destination node. WSNs have many nodes between the source and destination. Some nodes may be cooperative or non cooperative node. In Fig. 3, the nodes between source-to-intermediate nodes and intermediate node-to-the destination is used in cooperative communication, are called cooperative nodes. In networking operations from the source to the destination node, 9 nodes are involved.

\section{Energy consumption analysis of multihop MIMO technique}

Total power consumption in Transmitter circuit is given by (1)

$P_{T}=N_{T}\left(P_{D A C}+P_{\text {mix }}+P_{\text {filt }}\right)+P_{\text {syns }}$

where the number of transmitting nodes, digital to analog converter, mixer, transmitter filter and frequency (a)

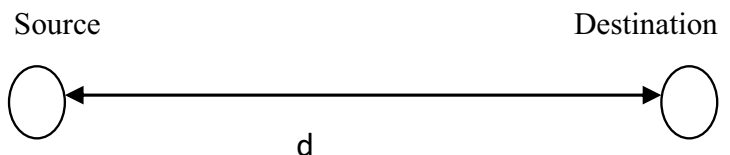

(b)

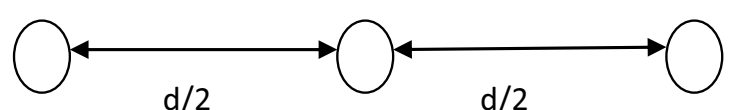

(c)

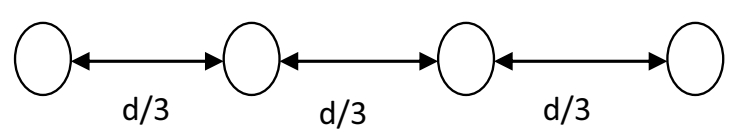

Fig. 1 Transmission distances for a 1 hop, b 2 hop, c 3 hop WSN

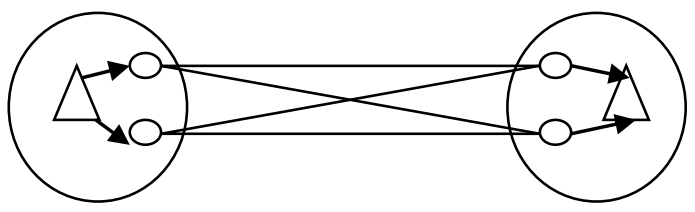

(a) $2 \times 2 \mathrm{~V}-\mathrm{MIMO}$
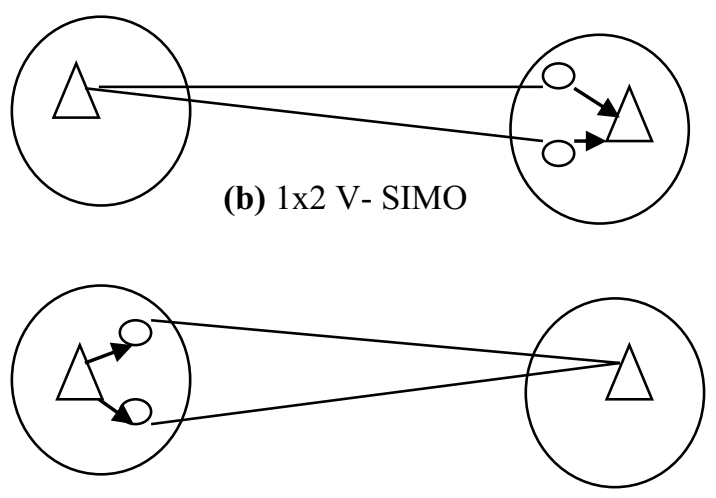

(c) $2 \times 1$ V-MISO

Fig. 2 Network topology for a $2 \times 2$ V-MIMO, b $1 \times 2$ V-SIMO, c $2 \times 1 \mathrm{~V}$-MISO techniques

synthesizer are denoted by $\mathrm{N}_{\mathrm{T}}, \mathrm{P}_{\mathrm{DAC}}, \mathrm{P}_{\text {mix }}, \mathrm{P}_{\text {filt }}$ and $\mathrm{P}_{\text {Syns }}$ respectively. Total power consumption in receiver circuit is given by (2)

$P_{R}=N_{R}\left(P_{A D C}+P_{\text {mix }}+P_{\text {filr }}+P_{\text {LNA }}+P_{\text {IFA }}\right)+P_{\text {Syns }}$

where number of receiving nodes, analog to digital converter, mixer, receiver filter, low noise amplifier, intermediate frequency amplifier and frequency synthesizer are denoted by $\mathrm{N}_{\mathrm{R}}, \mathrm{P}_{\mathrm{ADC}}, \mathrm{P}_{\text {mix }}, \mathrm{P}_{\text {filr }}, \mathrm{P}_{\mathrm{LNA}}, \mathrm{P}_{\mathrm{IFA}}$ and $\mathrm{P}_{\text {Syns }}$ respectively. The power amplifier power consumption $\left(P_{P A}\right)$ can be expressed as (3)
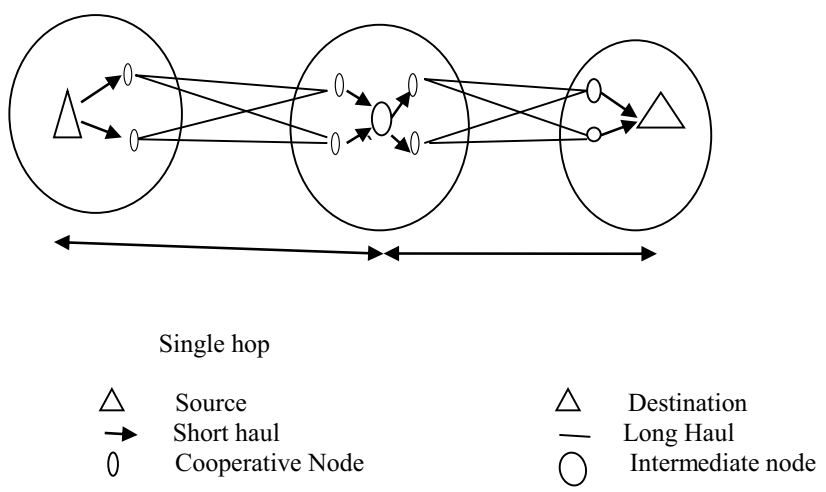

Fig. 3 2-Hop V-MIMO technique for energy efficient wireless sensor network 
$\mathrm{P}_{\mathrm{PA}}=(1+\alpha) \mathrm{P}_{\text {out }}$

$\alpha$ is a ratio related to the drain efficiency and $\mathrm{P}_{\text {out }}$ is the output of the amplifier. In Fig. 2a, the energy consumption for transmitting a bit in intracluster communication at transmitter side is given by [11]

$E_{\text {Ta-intra }}=\frac{(1+\alpha) E_{b}(4 \pi)^{2} d_{1}^{k} M_{l} N_{f}}{G_{t} G_{r} \lambda^{2}}+\frac{P_{T}}{R_{b}}+\frac{N_{R} P_{R}}{R_{b}}$

In Fig. 2a, the energy consumption for transmitting a bit in intracluster communication at receiver side is given by [11]

$E_{\text {Ra-intra }}=\frac{(1+\alpha) E_{b}(4 \pi)^{2} d_{1}^{k} M_{l} N_{f}}{G_{t} G_{r} \lambda^{2}}+\frac{N_{T} P_{T}}{R_{b}}+\frac{P_{R}}{R_{b}}$

In Fig. 2a, the energy consumption for transmitting a bit in intercluster communication is given by [11]

$E_{a-\text { inter }}=\frac{(1+\alpha) E_{b}(4 \pi)^{2} d_{L}^{k} M_{l} N_{f}}{G_{t} G_{r} \lambda^{2}}+\frac{N_{T} P_{T}}{R_{b}}+\frac{N_{R} P_{R}}{R_{b}}$

where $M_{l}, N_{f}, G_{t}, G_{r}, k, R_{b}$ and $\lambda$ are the link margin, receiver noise figure, transmit and receive antennas gain, path loss exponent, bit rate and wavelength respectively. $E_{b}$ denotes energy per bit. $d_{\perp}$ and $d_{\llcorner}$are the distances between transmitting and receiving nodes in intra and inter cluster communication, respectively. $E_{b}$ can be determined by the following Eq. (7)

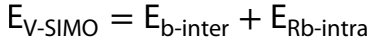

$E_{V-M I S O}=E_{T C-\text { intra }}+E_{C \_ \text {inter }}$

If $\mathrm{P}_{1}, \mathrm{P}_{2}, \mathrm{P}_{3}, \ldots \mathrm{P}_{\mathrm{n}}$ are single, double, triple hop and $\mathrm{n}$ hop transmitted power in SISO communication. Receiver requires $\mathrm{P}_{\mathrm{R}}$ power in SISO communication. Therefore, totals transmitted power in single-hop $\left(\left(\mathrm{P}_{1 \text { hop }}\right)\right.$, double-hop $\left(\mathrm{P}_{2 \text { hop }}\right)$, triple-hop $\left(\mathrm{P}_{\text {3hop }}\right)$ and $\mathrm{n}$-hop $\left(\mathrm{P}_{\text {nhop }}\right)$ SISO communication will be (11)

$P_{\text {hop }}=P_{1}+P_{R}$

where $\mathrm{P}_{1}=\mathrm{P}_{\mathrm{T}}+\mathrm{P}_{\mathrm{PA}}$ and $\mathrm{N}_{\mathrm{T}}=1, \mathrm{~N}_{\mathrm{R}}=1$

$P_{2 h o p}=2\left(\frac{P_{1}}{2^{k}}+P_{R}\right)$

$P_{3 h o p}=3\left(\frac{P_{1}}{3^{k}}+P_{R}\right)$

$P_{\text {nhop }}=n\left(\frac{P_{1}}{n^{k}}+P_{R}\right)$

Energy Consumption per bit for n-hop $2 \times 2$ V-MIMO transmissions is given as (15)

$E_{\text {nbt_MIMO }}=n\left(\frac{P_{\text {MIMO }}}{n^{k}}+R_{\text {MIMO }}\right)$

$\overline{\mathrm{P}}_{\mathrm{b}}=\frac{4}{\mathrm{~b}}\left(1-\frac{1}{2^{\frac{b}{2}}}\right) \frac{1}{2^{\mathrm{N}_{\mathrm{T}} \mathrm{N}_{\mathrm{R}}}}\left(1-\frac{1}{\sqrt{1+\frac{2 \mathrm{~N}_{0}}{\mathrm{E}_{\mathrm{b}}}}}\right)^{\mathrm{N}_{\mathrm{T}} \mathrm{N}_{\mathrm{R}}} \times \sum_{\mathrm{k}=0}^{\mathrm{N}_{\mathrm{T}} \mathrm{N}_{\mathrm{R}}-1} \frac{1}{2^{\mathrm{k}}}\left(\begin{array}{c}\mathrm{N}_{\mathrm{T}} \mathrm{N}_{\mathrm{R}}-1+\mathrm{k} \\ \mathrm{k}\end{array}\right) \times\left(1+\frac{1}{\sqrt{1+\frac{2 \mathrm{~N}_{0}}{\mathrm{E}_{\mathrm{b}}}}}\right)^{\mathrm{k}}$

where $\mathrm{N}_{\mathrm{O}}$ denotes the power spectral density, $\overline{\mathrm{P}}_{\mathrm{b}}$ denotes the BER and $b$ denotes constellation size. Now, in the Fig. 2a, the total energy for transmitting a bit using V-MIMO communication in a single hop is expressed as [12]

\section{Reliability of WSN}

WSN generally uses frequencies of $470 \mathrm{MHz}$ to $2.4 \mathrm{GHz}$. The single or multihop sensor network is used in environmental monitoring. Homogeneous sensor nodes are used

$$
\begin{aligned}
& E_{V-M I M O}=E_{T a-i n t r a}+E_{a-i n t e r}+E_{R a-i n t r a} \\
& \quad=\left[\frac{2(1+\alpha) E_{b}(4 \pi)^{2} d_{l}^{k} M_{1} N_{f}}{G_{t} G_{r} \lambda^{2}}+\frac{(1+\alpha) E_{b}(4 \pi)^{2} d_{L}^{k} M_{l} N_{f}}{G_{t} G_{r} \lambda^{2}}+\frac{5 P_{T}}{R_{b}}\right]+\left[\frac{5 P_{R}}{R_{b}}\right]
\end{aligned}
$$

$=[$ Total energy for transmitting a bit in single hop communication $\left.\left(\mathrm{P}_{\mathrm{MIMO}}\right)\right]+[$ Total energy for receiving a bit in single hop communication $\left(\mathrm{R}_{\mathrm{MIMO}}\right)$ ].

Now, in Fig. 2b, 2c, the total energy for transmitting a bit using V-SIMO and V-MISO communication in a single hop is given by (9) and (10) for this application and range of multihop network is up to $500 \mathrm{~m}$. The initial energy of each node is $1 \mathrm{~J}$. Generally, frequency is inversely proportional to radio range, but the disadvantages of lower frequency are the bigger size of the antenna [15]. If $P$ is the probability of a message delivered from one node to another node. Then, $\mathrm{P}^{\mathrm{n}}$ is the 
probability of successful delivery of a message transmitted over $\mathrm{n}$ hops. Therefore, multihop communication protocol is fault tolerant. Hence, it should be able to re-transmit lost packets. Network connectivity chooses whether multi-hop paths exist or not between any pair of sensor nodes. It is determined by node density, node distribution, effective communication radius and channel propagation model. Network connectivity is measured by probability of existence of n-hop route between sources-to-destination in the WSN [16]. Obviously, the probability of existence is inversely proportional to hop count.

Reliability is defined as, the success probability of n-hop route between source-to-destination in WSN. In multihop transmission, node will not fail even if link fails. If channel loss rate is e and a packet is transmitted from the source node to the base station using $\mathrm{n}$ time hoping. Then, the probability of a packet to reach sink node is given by [17]

$P=(1-e)^{n}$

If source sensor node transmits $L$ packets, then the probability of sink sensor node successfully receive $L$ packets can be expressed as (14)

$P=(1-e)^{n L}$

\subsection{Routing mechanism of multihop transmission}

Source sensor node broadcast a SRD packet. The relay sensor node forward the packets as long as they receive it. The destination sensor node instantly acknowledges a routing response packet (DRS) to source node, when it successfully receive the SRD packet from the source node.SRD packet and DRS Packet both follow the same route. After receiving the DRS packet by source nodes, routing procedure finishes.

The method of multihop routing procedure is shown in Fig. 4. The shortest path is applied in routing mechanism.

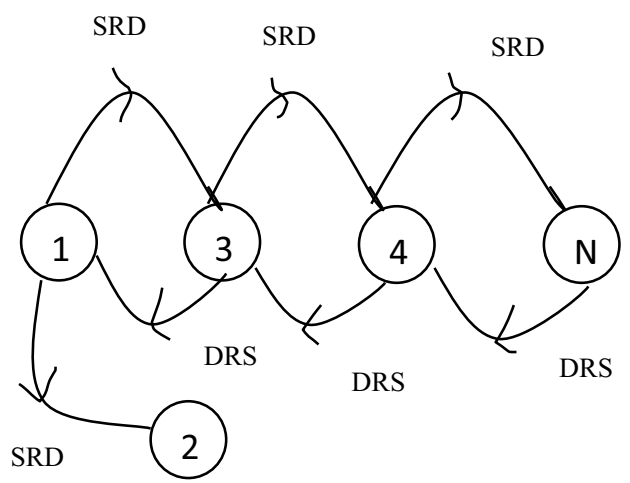

Fig. 4 The process of Multihop routing discovery
Thus, the relay sensor node which is closest to destination sensor node has the highest priority to transmit DRS packets.SRD and DRS packets may lose several times during packet transmission. If multihop routing discovery process fails, the data transmission between source and destination sensor nodes is not possible.

\section{Simulation result and discussion}

Figure 5 reveals the graph between the energy consumption per bit ( $\mathrm{mJ}$ ) and distance $(\mathrm{m})$ for various fixed hops. From the Fig. 5, it proves that the single hop uses less energy for short distance communication, whereas multiple hops become energy efficient for long distance communication. However, for all types of hops, energy consumption increases with an increase in transmission distance. Thus, if an optimal hop is chosen, the total energy consumption can be minimized through the SISO technique in the WSN.

Figure 6 shows the energy consumption analysis of SISO, $1 \times 2$ V-SIMO and $2 \times 2 \mathrm{~V}$-MIMO techniques for single hop communication. It indicates that for short distance communication, SISO technique consumes the lowest energy among all. For mid-range communication, V-SIMO technique is preferable as they consume the lowest energy among their all counterparts. Finally, for long distance communication, V-MIMO technique consumes the lowest energy among all [2]. This is because

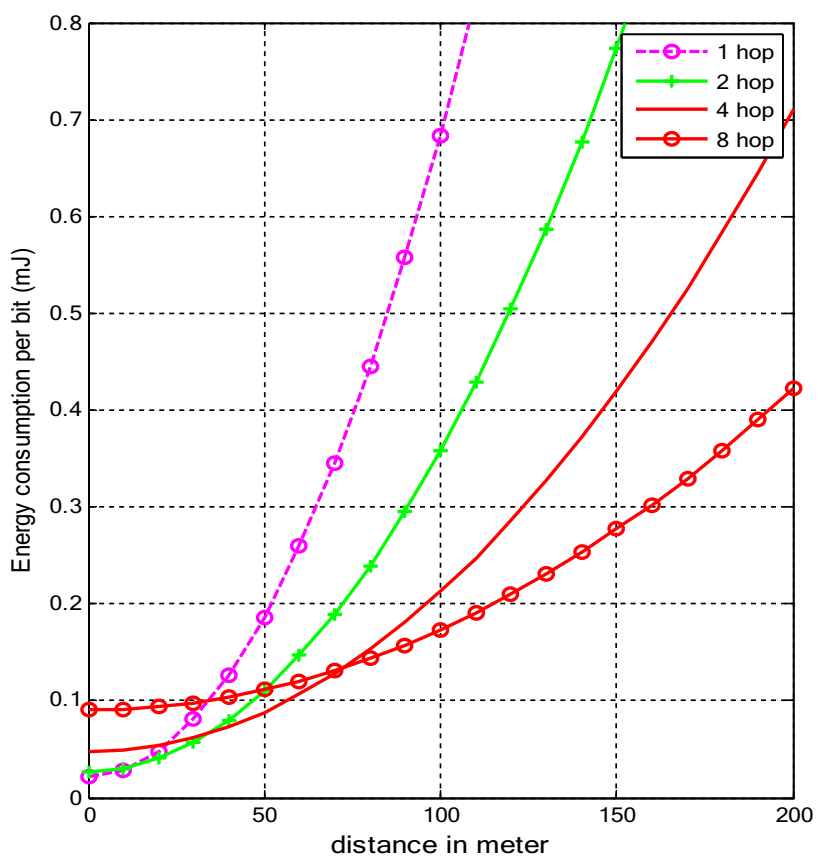

Fig. 5 Comparison of Energy consumption per bit of various multihop communications 


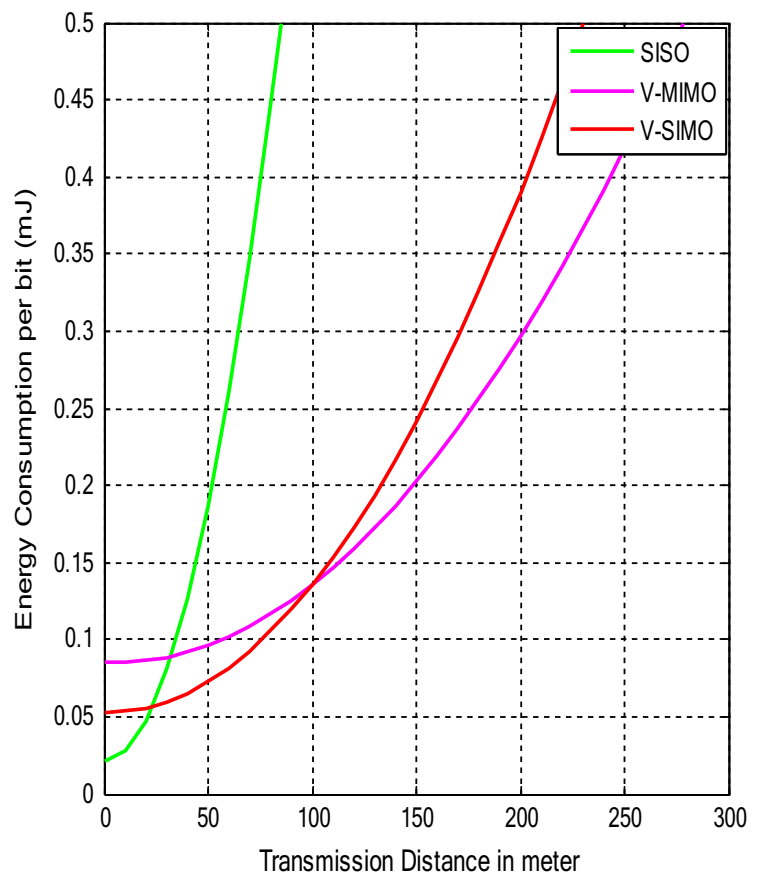

Fig. 6 Comparison of Energy consumption of various V-MIMO configurations

for short-distance communication, circuit energy dominant over transmission energy, whereas for long distance communication, transmission energy dominant over circuit energy. In each hop, the minimum energy consumption V-MIMO configuration (e.g., SISO, $2 \times 1 \mathrm{~V}$-MISO, $1 \times 2 \mathrm{~V}$-SIMO, $2 \times 2 \mathrm{~V}$-MIMO etc.) is needed.

Figure 7 depicts the energy consumption per bit versus hop count for distances 200 and $300 \mathrm{~m}$ for SISO technique. It can be observed that the total energy consumption shows a convex behavior. One can observe from Fig. 7 that energy consumption is lowest for 8 hops at distance $200 \mathrm{~m}$, and thus 8 hops is the most energy efficient at a distance of $200 \mathrm{~m}$ for the SISO technique. From the Fig. 7, it is evident that 10 hops are the optimum hops at distance $300 \mathrm{~m}$ for the SISO technique [9]. Using 8 hops and 10 hops for distance $200 \mathrm{~m}$ and $300 \mathrm{~m}$ are not practically suitable. Thus, SISO technique is good for short range communication in the WSN already prove in Fig. 6.

Figure 8 depicts the energy consumption per bit versus number of hops for distances 200 and $300 \mathrm{~m}$ for $2 \times 2$-MIMO technique. One can observe from Fig. 8 , that energy consumption is lowest for 2 hops at distance $200 \mathrm{~m}$, and thus 2 hops is the most energy efficient at a distance of $200 \mathrm{~m}$ for the $2 \times 2 \mathrm{~V}$-MIMO technique. From Fig. 8 , it is evident that 3 hops are the optimum hops at distance $300 \mathrm{~m}$ for the $2 \times 2 \mathrm{~V}$-MIMO technique [9]. Thus, the energy saving for long range communication in WSN

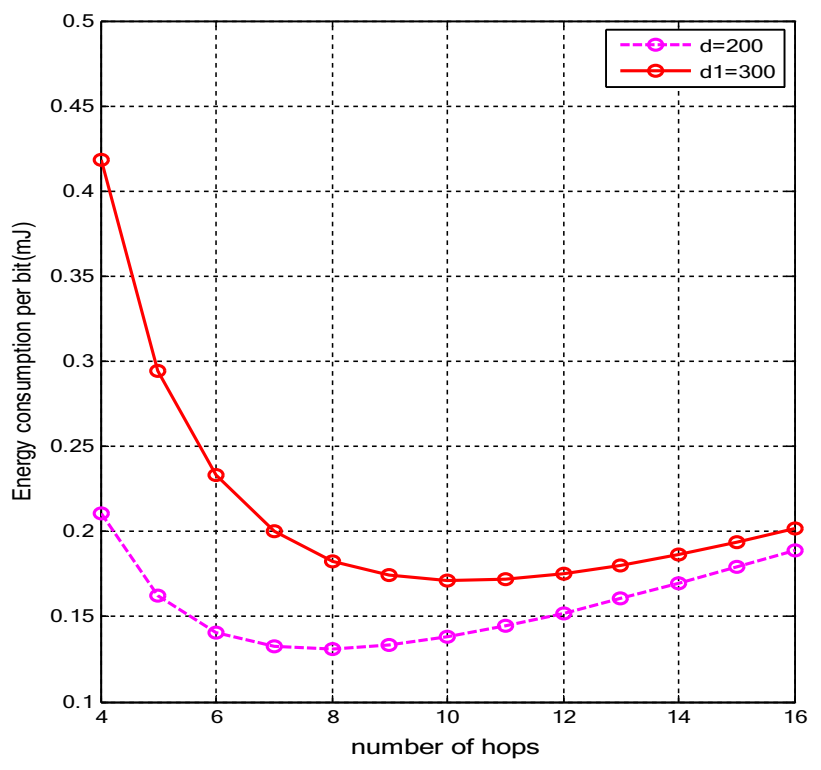

Fig. 7 Energy consumption per bit versus number of hops at distances 200 and $300 \mathrm{~m}$ for SISO technique

can be obtained through the selection of optimum hop MIMO technique.

In Fig. 9, energy consumption per bit of SISO, 3 hop SISO, $2 \times 2$ V-MIMO, 2-hop V-MIMO and 3-hop V-MIMO communication techniques are compared up to some fixed distance. The simulation result indicates that there is



Fig. 8 Energy consumption per bit versus number of hops at distances 200 and $300 \mathrm{~m}$ for $2 \times 2 \mathrm{~V}$-MIMO technique 
Fig. 9 The Comparison of energy consumption of SISO, 3 hop SISO, $2 \times 2$ V-MIMO, 2 hop V-MIMO, 3 hop V-MIMO techniques

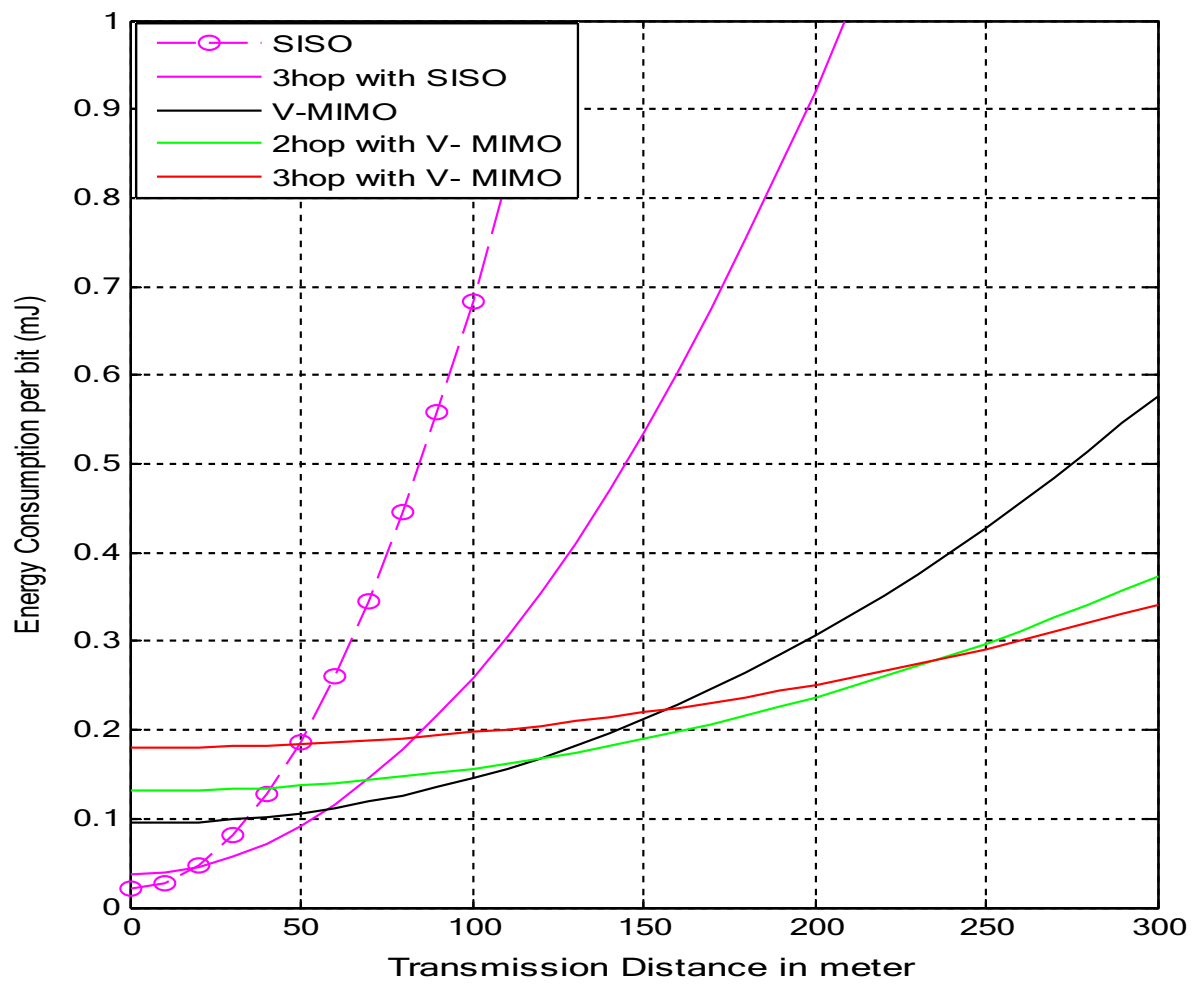

no need of multihop communication at smaller distances. The SISO technique is suitable for a small distance up to $30 \mathrm{~m}$, whereas $\mathrm{V}-\mathrm{MIMO}$ is energy efficient for mid range communication $(\mathrm{d}<120 \mathrm{~m})$. For long distance communication $(\mathrm{d}>120 \mathrm{~m})$, the proposed technique is more energy efficient than SISO, $2 \times 2$ V-MIMO, 3 hop SISO techniques $[11,18,19]$. Therefore, 2 hop V-MIMO technique is more energy efficient than other techniques at the $d=200 \mathrm{~m}$, while 3 hop V-MIMO technique is more energy efficient than other techniques at the $\mathrm{d}=300 \mathrm{~m}$. It is shown in Table $1[11,18,19]$. The reason is that more number of hops is needed as communication distance increases, and MIMO technique is more energy efficient than SISO technique for long range communication in the WSN.
The path loss exponent $\mathrm{n}$ depends on propagation environment. In free space, the value of the path loss exponent ( $k$ ) is equal to 2 and when obstacle exists then $k$ will have a bigger value. The reference path loss determines using the free space path loss formula. The effect of path loss exponent on energy consumption can be determined in different environments (Table 2).

Figures 10 and 11 show the energy consumption of single hop, double hop, triple hop, quad hop network for different value of path loss exponent. Figure 10 shows that single hop is more energy efficient than multihop communication for short distance communication $(\mathrm{d}=15 \mathrm{~m})$ and low value path loss exponent $(k=2)$, while Fig. 11 shows that multihop communication is more energy efficient

Table 1 Comparison of energy consumption per bit for different communication techniques such as SISO, 3 hop SISO, $2 \times 2$ V-MIMO, 2-hop V-MIMO and 3-hop V-MIMO

\begin{tabular}{lllllllll}
\hline $\begin{array}{l}\text { Distance } \\
\text { in meters }\end{array}$ & \multicolumn{2}{l}{ Energy consumption per bit in $\mathrm{mJ}$} \\
\cline { 2 - 8 } & SISO (This work) & $\begin{array}{l}3 \text { hop SISO } \\
\text { (This work) }\end{array}$ & $\begin{array}{l}2 \times 2 \text { V-MIMO } \\
{[11]}\end{array}$ & $\begin{array}{l}2 \times 2 \text { V-MIMO } \\
{[18]}\end{array}$ & $\begin{array}{l}2 \times 2 \text { V-MIMO } \\
{[19]}\end{array}$ & $\begin{array}{l}2 \times 2 \text { V-MIMO } \\
\text { (This work) }\end{array}$ & $\begin{array}{l}2 \text { hop } \\
\text { V-MIMO } \\
\text { (Proposed) }\end{array}$ & $\begin{array}{l}3 \text { hop } \\
\text { (Proposed) }\end{array}$ \\
\hline 20 & 0.048 & 0.048 & - & 0.298 & 0.125 & 0.097 & 0.132 & 0.181 \\
30 & 0.098 & 0.099 & - & 0.336 & 0.127 & 0.098 & 0.133 & 0.182 \\
100 & 0.684 & 0.257 & 0.545 & 0.367 & 0.206 & 0.146 & 0.157 & 0.197 \\
200 & 2.671 & 0.921 & 4.383 & 0.463 & 1.272 & 0.307 & 0.237 & 0.251 \\
300 & 5.99 & 2.025 & - & - & - & 0.578 & 0.373 & 0.341 \\
\hline
\end{tabular}


Table 2 Path loss exponents for different environments

\begin{tabular}{lll}
\hline S. no. & Environment & $\begin{array}{l}\text { Path loss } \\
\text { exponent } \\
(\mathrm{k})\end{array}$ \\
\hline 1 & Free space & 2 \\
2 & Urban area & $2.7-3.5$ \\
3 & Obstructed in factories & $2-3$ \\
4 & In building line-of-sight & $1.6-1.8$ \\
\hline
\end{tabular}

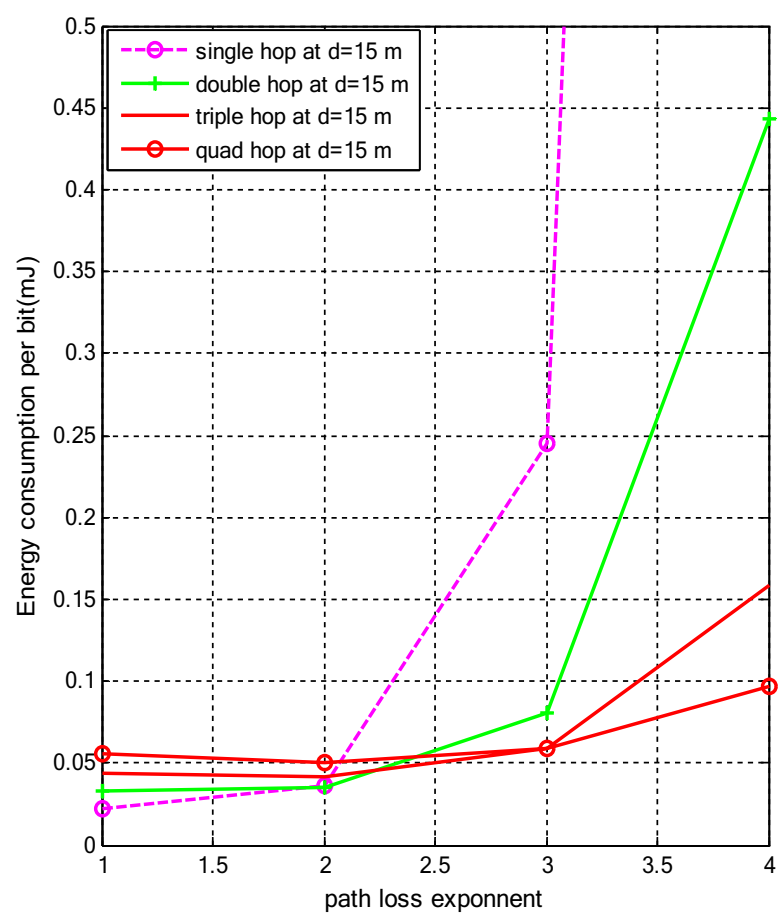

Fig. 10 Energy consumption per bit versus path loss exponent for SISO technique at various hop at $\mathrm{d}=15 \mathrm{~m}$

than single hop communication for long distance communication $(\mathrm{d}=75 \mathrm{~m})$ and low value path loss exponent $(k=2)$ in the WSN. For both short and long-range communication, energy consumption increases with the increase in the path loss exponent in the single as well as multihop communication [14]. Figures 10 and 11 indicate that after path loss exponent $(k)=2$, energy consumption increases more rapidly in long distance communication than short distance communication in both the single hop and multihop communication in the WSN.

Figure 12 indicates the energy consumption versus path loss exponent of SISO and MIMO techniques for short distance communication. Figure 12 shows that SISO technique is more energy efficient than $2 \times 2$ V-MIMO technique for short distance communication and low value of the path loss exponent. Figure 13 indicates the energy consumption versus path

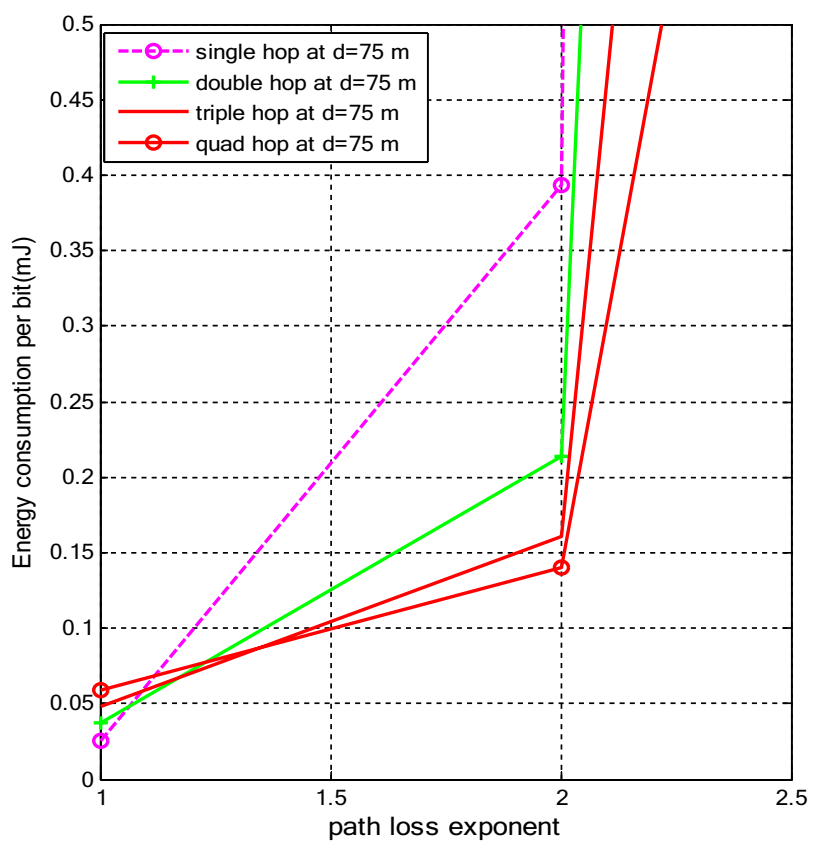

Fig. 11 Energy consumption versus path loss exponent for SISO technique at various hop at $d=75 \mathrm{~m}$

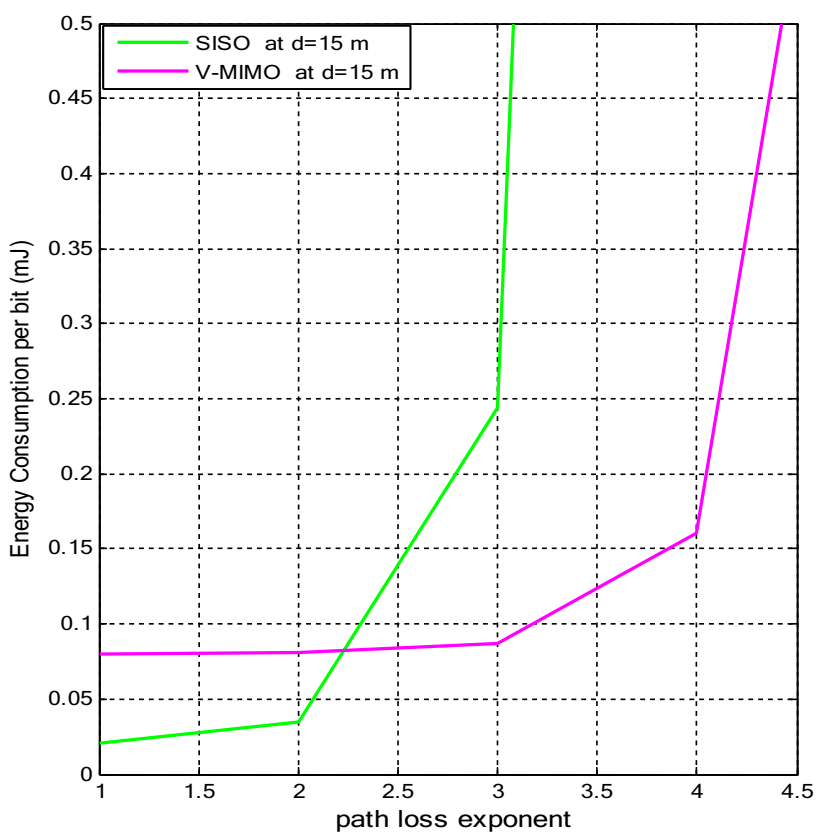

Fig. 12 Energy consumption per bit versus path loss exponent for SISO and V-MIMO technique at $d=15 \mathrm{~m}$

loss exponent of SISO and MIMO communication for long distance communication. Figure 13 shows that $2 \times 2 \mathrm{~V}$-MIMO technique is more energy efficient than SISO technique at path loss exponent $\mathrm{k}=2$ for long distance communication. 


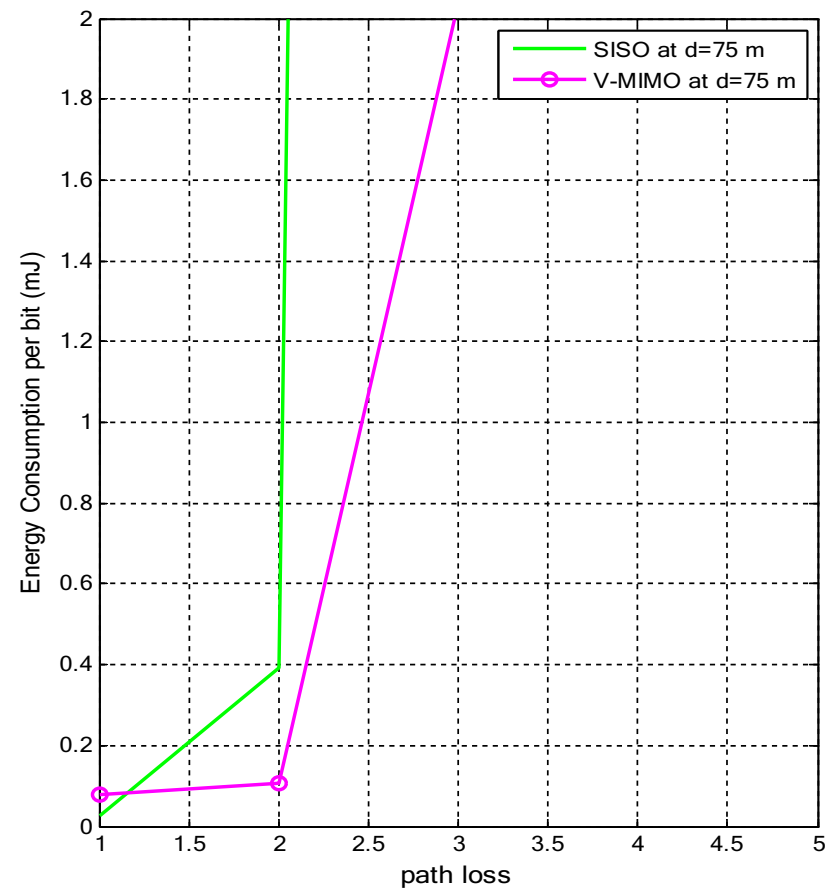

Fig. 13 Energy consumption per bit versus path loss exponent for SISO and V-MIMO technique at $d=75 \mathrm{~m}$

From Figs. 12 and 13, one can observe that Energy consumption is directly proportional to path loss exponent in both techniques [14]. But, energy consumption increase more sharply after path loss exponent $(k)=2$ in both SISO and V-MIMO techniques.

Figure 14 shows the reliability of multihop wireless sensor network. Figure 14 reveals that the reliability of multihop network is inversely proportional to the number of

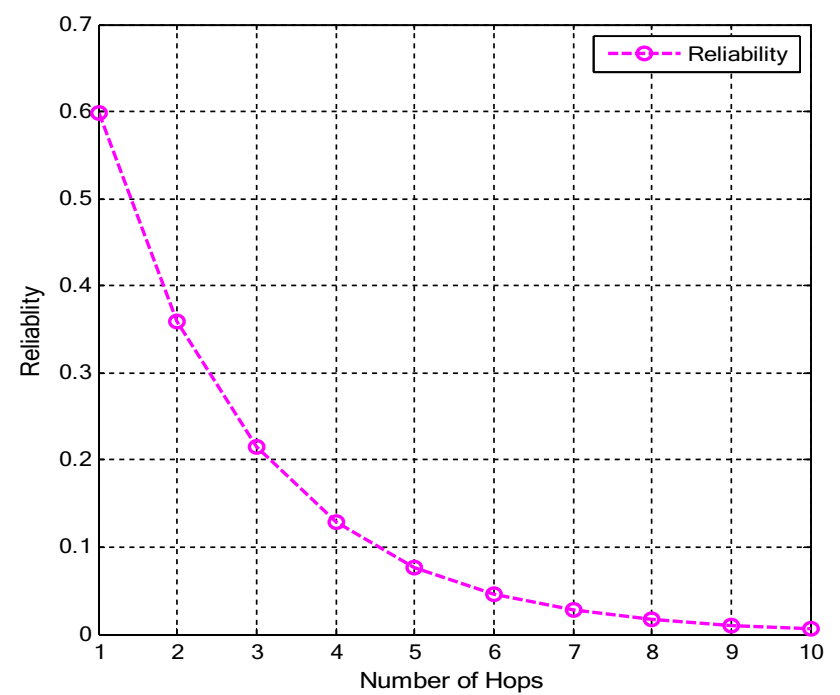

Fig. 14 Reliability of WSN hops because the probability of successful delivery of a message transmitted over $\mathrm{n}$ hops inversely proportional to the channel loss rate. Therefore, Reliability of the network increases with decrease in channel loss rate [17] (Table 3).

\section{Conclusion}

The multihop V-MIMO technique is proposed to design an energy efficient WSN for long distance communication. The multihop technique transmits the data packets from source to sink (BS) through various relay nodes, whereas V-MIMO technique improves the data rate through spatial multiplexing in the WSN. The simulation results prove that 2 hop V-MIMO technique is more energy efficient than other techniques at the $d=200 \mathrm{~m}$, while 3 hop V-MIMO technique is more energy efficient than other techniques at the $d=300 \mathrm{~m}$. The simulation results also prove that energy consumption is directly proportional to the path loss exponent in single and multihop communication. The results reveal that probability of successful delivery of a message transmitted from source to sink is inversely proportional to the number of hops. Various V-MIMO configurations (SISO, $1 \times 2$ SIMO, $2 \times 2 \mathrm{MIMO}$ etc.) can be combined with network coding, spatial modulation, etc. By appropriate combination of these techniques a very effective energy efficient multihop Virtual MIMO technique can be developed in the WSN.

Table 3 Simulation parameters

\begin{tabular}{ll}
\hline Symbol & Value \\
\hline$\lambda$ & $0.125 \mathrm{~m}$ \\
$a$ & 0.47 \\
$\mathrm{G}_{\mathrm{t}} \mathrm{G}_{\mathrm{r}}$ & $5 \mathrm{dBi}$ \\
$\mathrm{No}$ & $-174 \mathrm{dBm} / \mathrm{Hz}$ \\
$\mathrm{B}$ & $10 \mathrm{kHz}$ \\
$\mathrm{P}_{\text {mix }}$ & $30.3 \mathrm{~mW}$ \\
$\mathrm{P}_{\text {IFA }}$ & $2 \mathrm{~mW}$ \\
$\mathrm{P}_{\text {syn }}$ & $50 \mathrm{~mW}$ \\
$\mathrm{P}_{\text {filt }}$ & $2.5 \mathrm{~mW}$ \\
$\mathrm{P}_{\text {filr }}$ & $2.5 \mathrm{~mW}$ \\
$\mathrm{~N}_{\mathrm{f}}$ & $10 \mathrm{~dB}$ \\
$\mathrm{P}_{\text {LNA }}$ & $20 \mathrm{~mW}$ \\
$M_{\mathrm{l}}$ & $40 \mathrm{~dB}$ \\
$\mathrm{P}_{\text {DAC }}$ & $15.43 \mathrm{~mW}$ \\
$\mathrm{P}_{\text {ADC }}$ & $6.62 \mathrm{~mW}$ \\
$\mathrm{~L}$ & $10 \mathrm{bits}$ \\
$\mathrm{e}$ & 0.05 \\
\hline
\end{tabular}

SN Applied Sciences A SPRINGER NATURE journa 
Acknowledgements The authors have no relevant financial interests in the paper and no other potential conflicts of interest to disclose.

\section{Compliance with ethical standards}

Conflict of interest The authors declare that they have no conflict of interest.

\section{References}

1. Kumar SM, Intekhab AS, Akhtar IS, Kumar SV, Amit C (2018) A survey of wireless sensor network and its types. https://doi. org/10.1109/icacccn.2018.8748710

2. Cui S, Goldsmith AJ, Bahia A (2004) Energy-efficiency of MIMO and cooperative MIMO techniques in sensor networks. IEEE J Sel Areas Commun 22(6):1089-1098

3. Jayaweera SK (2006) Virtual MIMO-based cooperative communication for energy-constrained wireless sensor networks. IEEE Trans Wirel Commun 5(5):984-989

4. Priyadarshi R, Rawat $P$, Nath V (2019) Energy dependent cluster formation in heterogeneous wireless sensor network. Microsyst Technol 25:2313-2321

5. Ar-Reyouchi EM, Chatei Y, Ghoumid K, Hammouti M, Hajji B (2017) Efficient coding techniques algorithm for cluster-heads communication in wireless sensor networks. Int J Electron Commun: AEÜ 82:294-304

6. Priyadarshi R, Soni SK, Nath V (2018) Energy efficient cluster head formation in wireless sensor network. Microsyst Technol 24:4775-4784

7. Chung J-M, Kim J, Han D (2012) Multihop hybrid virtual MIMO scheme for wireless sensor networks. IEEE Trans Veh Technol 61(9):4069-4078

8. Kheireddine $M$, Abdellatif $R$ (2014) Analysis of hops length in wireless sensor networks. Wirel Sens Netw 6:109-117

9. Lambor SM, Joshi SM (2018) Optimal hops for minimal route power under SINR constraints in wireless sensor networks. IET Wirel Sens Syst 8(4):176-182
10. Singh MK, Amin SI (2020) Energy-efficient data transmission technique for wireless sensor networks based on DSC and virtual MIMO. ETRI J 42:341-350. https://doi.org/10.4218/etrij .2018-0632

11. Kanthimathi M., Amutha R., Senthil Kumar K. (2018). Energy Efficient Differential Cooperative MIMO Algorithm for Wireless Sensor Networks. Wireless Personal Communications, Issue 4

12. Liu X, Gong X, Zheng Y (2014) Reliable cooperative communications based on random network coding in multi-hop relay WSNs. IEEE Sens J 14(8):2514-2523

13. Islam MR, Kim J (2010) On the cooperative MIMO communication for energy-efficient cluster-to-cluster transmission at wireless sensor network. Ann Telecommun 65(5-6):325-340

14. Doddapaneni K et al (2012) Path loss effect on energy consumption in a WSN. In: 14th international conference on computer modelling and simulation, Cambridge, 2012, pp 569-574. https ://doi.org/10.1109/uksim.2012.87

15. Huebner $C$, Oliver RC, Hanelt $S$, Wagenknecht $T$, Monsalve $A$ (2013) Long-range wireless sensor networks with transmitonly nodes and software-defined receivers. Wirel Commun Mob Comput 13:1499-1510

16. Wenxing L, Muqing W, Min Z, Peizhe L, Tianze L (2017) Hop count limitation analysis in wireless multi-hop networks. Int J Distrib Sens Netw 13(1):1-13

17. Luan Z (2017) Calculation and simulation of transmission reliability in wireless sensor network based on network coding. iJOE 13(12):150-161

18. Islam MR, Han YS (2011) Cooperative MIMO communication at wireless sensor network: an error correcting code approach. Sensors 11:9887-9903. https://doi.org/10.3390/s111009887

19. Senthil K et al (2017) Energy efficient V-MIMO using turbo codes in wireless sensor networks. In: 2017 IEEE. 978-1-5090-6221-8/17/\$31.00_c

Publisher's Note Springer Nature remains neutral with regard to jurisdictional claims in published maps and institutional affiliations. 